\title{
Parametric Excitation of a Bose-Einstein Condensate: From Faraday Waves to Granulation
}

\author{
J. H. V. Nguyen, ${ }^{1}$ M. C. Tsatsos, ${ }^{2}$ D. Luo, ${ }^{1}$ A. U. J. Lode,${ }^{3,4,5}$ G. D. Telles, ${ }^{2}$ V. S. Bagnato, ${ }^{2}$ and R. G. Hulet ${ }^{1, *}$ \\ ${ }^{1}$ Department of Physics and Astronomy, Rice University, Houston, Texas 77005, USA \\ ${ }^{2}$ Institute of Physics of São Carlos, University of São Paulo, P.O. Box 369, \\ 13560-970, São Carlos, SP, Brazil \\ ${ }^{3}$ Wolfgang Pauli Institute clo Faculty of Mathematics, University of Vienna, \\ Oskar-Morgenstern Platz 1, 1090 Vienna, Austria \\ ${ }^{4}$ Vienna Center for Quantum Science and Technology, Atominstitut, TU Wien, \\ Stadionallee 2, 1020 Vienna, Austria \\ ${ }^{5}$ Department of Physics, University of Basel, Klingelbergstrasse 82, CH-4056 Basel, Switzerland
}

(Received 29 June 2018; revised manuscript received 28 December 2018; published 25 March 2019)

\begin{abstract}
We explore, both experimentally and theoretically, the response of an elongated Bose-Einstein condensate to modulated interactions. We identify two distinct regimes differing in modulation frequency and modulation strength. Longitudinal surface waves are generated either resonantly or parametrically for modulation frequencies near the radial trap frequency or twice the trap frequency, respectively. The dispersion of these waves, the latter being a Faraday wave, is well reproduced by a mean-field theory that accounts for the 3D nature of the elongated condensate. In contrast, in the regime of lower modulation frequencies, we find that no clear resonances occur, but with an increased modulation strength, the condensate forms an irregular granulated distribution that is outside the scope of a mean-field approach. We find that the granulated condensate is characterized by large quantum fluctuations and correlations, which are well described with single-shot simulations obtained from wave functions computed by a beyondmean-field theory at zero temperature, the multiconfigurational time-dependent Hartree for bosons method.

DOI: 10.1103/PhysRevX.9.011052

Subject Areas: Atomic and Molecular Physics, Quantum Physics
\end{abstract}

\section{INTRODUCTION}

Spatial patterns frequently emerge in driven fluids in a variety of contexts, including chemistry, biology, and nonlinear optics [1]. Instabilities in these systems can generally be categorized as Rayleigh-Bénard convection, Taylor-Couette flow, or parametric surface waves. One of the earliest and best-known examples of the latter type are the surface waves found by Faraday when a vessel containing a fluid is shaken vertically [2]. The resulting standing-wave patterns that appear on the fluid surface arise from the parametric excitation of collective modes of the fluid. The Faraday experiment has been repeated in various geometries where complex patterns were observed for small driving amplitudes [3]. Chaotic behavior, such as subharmonic bifurcation, is seen when the drive amplitude

\footnotetext{
* Corresponding author. randy@rice.edu.

Published by the American Physical Society under the terms of the Creative Commons Attribution 4.0 International license. Further distribution of this work must maintain attribution to the author(s) and the published article's title, journal citation, and DOI.
}

is strong [3-6], and this behavior has been connected to the onset of turbulence [7].

A model of the Faraday instability has been developed for an inviscid fluid in which the underlying hydrodynamic equations are linearized [8]. The linearized dynamics are described by a Mathieu equation, $\ddot{x}+p(t) x=0$, where $x$ is the displacement, $p(t)=\Omega^{2}[1+\epsilon \cos (\omega t)]$ is the drive, representing a parametrically driven (undamped) harmonic oscillator with a natural frequency $\Omega$, drive frequency $\omega$, and drive amplitude $\epsilon$. Solving the equations using a Floquet analysis results in a series of resonances at $\omega=2 \Omega / n$, where $n$ is an integer [9].

Superfluids are particularly interesting in the context of Faraday waves, because the damping of collective modes can be much smaller than in normal fluids and because patterns may dissipate by the formation of quantized vortices in two or three dimensions. Several theoretical works have investigated Faraday waves in Bose-Einstein condensates (BECs) of atomic gases [10-16]. To our knowledge, only three experiments on Faraday waves in superfluids have been performed, one in which a vessel containing liquid ${ }^{4} \mathrm{He}$ is vertically shaken in a way similar to the original Faraday experiment [17], a pioneering experiment in which Faraday waves were excited by modulation of the transverse trap frequency $\omega_{r}$ of an 
elongated BEC of $\mathrm{Rb}$ atoms [18], and another in which a nondestructive imaging technique is used to observe Faraday waves in a BEC of Na atoms [19]. In the BEC experiments, the transverse breathing mode, excited at a frequency of $2 \omega_{r}$, strongly couples to the density and, hence, to the nonlinear interactions of the condensate. This coupling produces the longitudinal sound waves responsible for creating Faraday waves $[18,19]$. The spatial period of the Faraday waves was measured as a function of $\omega$, and the response to the strength $\epsilon$ of the drive was investigated [18]. In a related BEC experiment, modulation of the scattering length in a regime of large modulation amplitude and frequency resulted in the stimulated emission of matterwave jets from a two-dimensional BEC of Cs atoms [20].

In this paper, we report measurements characterizing the response of an elongated $\mathrm{BEC}$ to direct modulation of the interaction parameter using a Feshbach resonance [21-23]. For drive frequencies near the first parametric resonance ( $\omega$ near $2 \omega_{r}$ ), we observe robust linear spatial patterns characterized by a spatial period $\lambda_{F}(\omega)$ consistent with Faraday waves. We also observe the response of the gas to the nextlowest "resonant" mode $\left(\omega\right.$ near $\left.\omega_{r}\right)$ [15]. We have also investigated how $\lambda_{F}$ depends on the interaction strength. These measurements are compared with a theory that fully incorporates radial, as well as axial, dynamics using a variational method [15], and, as we show, the agreement is excellent.

Furthermore, we also explore a different modulation regime, both experimentally and theoretically, where $\omega$ is far from any trap frequency. The behavior in this regime is distinctly different; no clear resonances are observed, and much larger modulation amplitude and time are needed to obtain a significant response. The response is not regular in this regime, and no clear patterns emerge; rather, modulation produces a series of irregular grains.

Granulation is found in a variety of systems extending over many length and energy scales [24,25]. In quantum gases, granular states have been discussed previously in the context of perturbed atomic BECs and explored theoretically using a mean-field approach [26,27]. Granular states have been defined to have the following properties [26]:

(i) They are dynamical quantum states where particles cluster in higher-density grains interleaved by regions of very low density, (ii) the spatial distribution of grains is random, and (iii) the grain size is variable and of a multiscale nature.

Our theoretical description uses the multiconfigurational time-dependent Hartree method for bosons (MCTDHB or MB) $[28,29]$. The MCTDHB captures many of the salient experimental observations and goes systematically beyond a mean-field description obtained from the Gross-Pitaevskii equation. The discrepancies between the Gross-Pitaevskii mean-field description and both the experimental observations and our MCTDHB results hint that granulation emerges concurrently with many-body correlations.

\section{FARADAY WAVES}

In our experiment, we confine a gas of up to $8 \times 10^{5}{ }^{7} \mathrm{Li}$ atoms in a single-beam optical dipole trap and cool them to well below $T_{c}=330 \mathrm{nK}$, the transition temperature for Bose-Einstein condensation [22]. This configuration results in a highly elongated cylindrical trapping geometry whose corresponding axial and radial harmonic frequencies are $\omega_{z}=(2 \pi) 7 \mathrm{~Hz}$ and $\omega_{r}=(2 \pi) 475 \mathrm{~Hz}$, respectively. The atoms are optically pumped into the lowest ground-state hyperfine level $\left|F=1, m_{F}=1\right\rangle$, where their $s$-wave scattering length may be controlled using a broad Feshbach resonance located at $737.7 \mathrm{G}$ [30-33]. The magnetic field is sinusoidally modulated according to $B(t)=\bar{B}+\Delta B \sin (\omega t)$, resulting in a modulated scattering length $a(t)$. The modulation amplitude $\Delta B$, modulation time $t_{m}$, and hold time $t_{h}$ following $t_{m}$ are varied for each value of the modulation frequency $\omega$, as necessary to produce a Faraday pattern with similar contrast. After $t_{h}$, we take a polarization phase-contrast image [34] with a probe laser propagating along the $x$ axis, perpendicular to the cylindrical $z$ axis of the trap. These images provide column-density distributions that we integrate along the $y$ axis to obtain line-density profiles. We apply a fast-Fourier transform (FFT) to these profiles in order to determine the spectrum of spatial frequencies exhibited by the BEC following modulation.

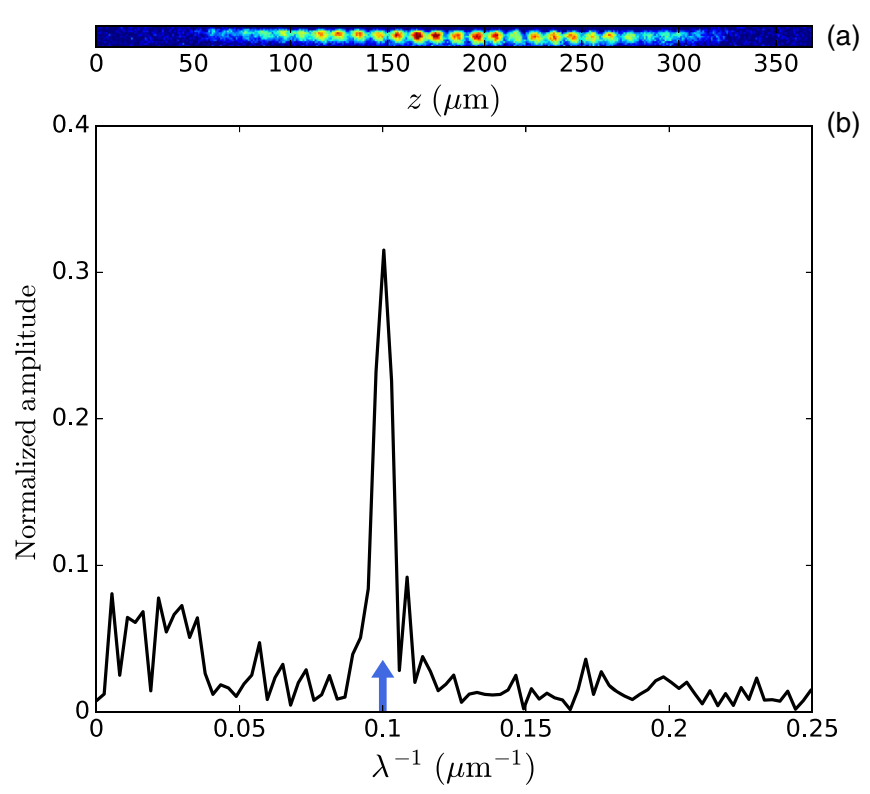

FIG. 1. (a) Column-density image; (b) FFT of the line density. The modulation parameters are $\omega=(2 \pi) 950 \mathrm{~Hz}, \bar{B}=572.5 \mathrm{G}$, $\Delta B=5 \mathrm{G}$, corresponding to a mean scattering length $\bar{a}=4.2 a_{0}$, and a modulation amplitude $\Delta a=0.9 a_{0}$, where $a_{0}$ is the Bohr radius. In addition, $t_{m}=5 \mathrm{~ms}$ and $t_{h}=20 \mathrm{~ms}$. The blue arrow indicates the calculated $\lambda_{F}^{-1}$ for these parameters. The dc component is subtracted, and the FFT amplitude is normalized by this dc value. 
A typical image of a single experimental run is shown in Fig. 1(a). In this example, $\omega=(2 \pi) 950 \mathrm{~Hz}$ is resonant with the Faraday mode at $\omega=2 \omega_{r}$. A surface wave is generated after $t_{m}=5 \mathrm{~ms}$ of modulation followed by $t_{h}=20 \mathrm{~ms}$. The FFT, shown in Fig. 1(b), features a single dominant peak corresponding to a spatial period of $\lambda=10 \mu \mathrm{m}$.

Figure 2 shows the spatial period of the observed structure as a function of $\omega$. Typically, $t_{h}=0 \mathrm{~ms}$ and $20<t_{m}<40 \mathrm{~ms}$, with the exception of $\omega=\omega_{r}$ and $\omega=2 \omega_{r}$. Near these resonances, the modulation time was kept short, $t_{m}=20 \mathrm{~ms}$ and $t_{m}=5 \mathrm{~ms}$, respectively, followed by $t_{h}=20 \mathrm{~ms}$. The blue data points correspond to the spatial period of the primary peak in the FFT spectrum. Except for the point at $\omega=(2 \pi) 475 \mathrm{~Hz}$, the period monotonically increases with decreasing $\omega$. The blue line in Fig. 2 is the result of a 3D variational calculation of $\lambda_{F}$ [15], which fits the data well. We verified that the standingwave surface-wave amplitude oscillates at $\omega / 2$ for $\omega$ near $2 \omega_{r}$, consistent with its identification as a Faraday wave, which is excited parametrically.

The excitation at $\omega=(2 \pi) 475 \mathrm{~Hz}=\omega_{r}$ is not a subharmonic of the Faraday mode at $2 \omega_{r}$ but rather the nextlowest mode in the infinite series of modes, identified as the

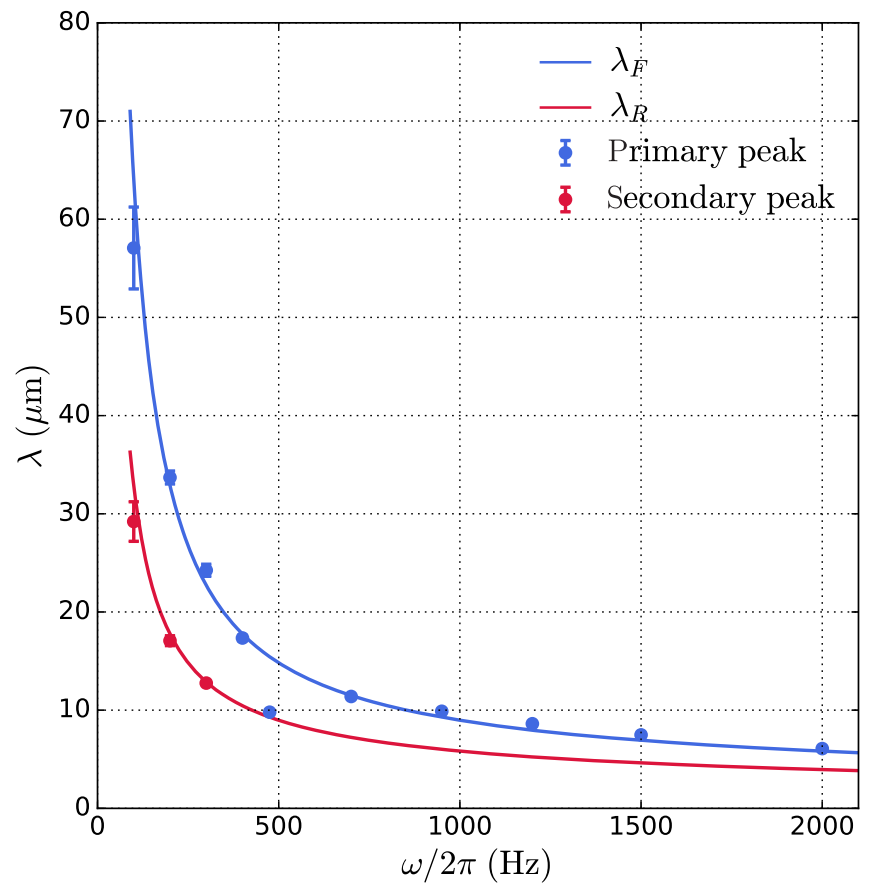

FIG. 2. Spatial period vs $\omega$. The blue data points are the primary peak of the FFTs, while the red data points correspond to a secondary peak, where one exists. The error bars here, and in each subsequent figure, correspond to the standard error of the mean determined from ten different experimental runs for each point. The solid blue line is the calculated spatial period $\lambda_{F}$ of the Faraday mode, while the red is that of the resonant mode $\lambda_{R}$ [15]. The resonant mode dominates only when $\omega$ is tuned to resonance at $\omega_{r}$, producing the observed primary peak. "resonant" mode in Ref. [15]. In addition to having a different dispersion relation, this mode is also weaker, and therefore more difficult to excite, except exactly on resonance, $\omega=\omega_{r}$, where the growth rate of the resonant mode exceeds that of the Faraday mode [15]. A similar excitation at $\omega_{r}$ was previously reported [18]. The theoretical calculation of the period of this mode is indicated in Fig. 2 by the red line, $\lambda_{R}$ [15].

We find that, as $\omega$ is tuned away from $2 \omega_{r}=$ $(2 \pi) 950 \mathrm{~Hz}$, a larger modulation amplitude $\Delta B$ and modulation time $t_{m}$ are required to obtain a pattern with similar contrast. For example, Fig. 3 displays the spectrum for $\omega=(2 \pi) 200 \mathrm{~Hz}$, for which $\Delta B=35 \mathrm{G}, t_{m}=20 \mathrm{~ms}$, and $t_{h}=20 \mathrm{~ms}$. Two peaks dominate the spectrum: the primary peak at a lower spatial frequency and a secondary peak at roughly twice this spatial frequency. These secondary peaks appear only for $\omega \lesssim(2 \pi) 400 \mathrm{~Hz}$ and are identified by the red data points in Fig. 2. The appearance of the nextlowest mode depends on being sufficiently near its resonance frequency at $\omega=\omega_{r}$ and far enough off resonant with the Faraday mode at $\omega=2 \omega_{r}$ that it does not dominate the FFT spectrum. We have looked for additional modes in the data, but the FFT spectrum is dominated by the off-resonant response to the $2 \omega_{r}$ and $\omega_{r}$ resonances, and we are unable to observe any resonances below $\omega_{r}$. A comparison of the period of these secondary peaks with the theoretically calculated solid red line indicates that they correspond to the resonant mode $\lambda_{R}$.

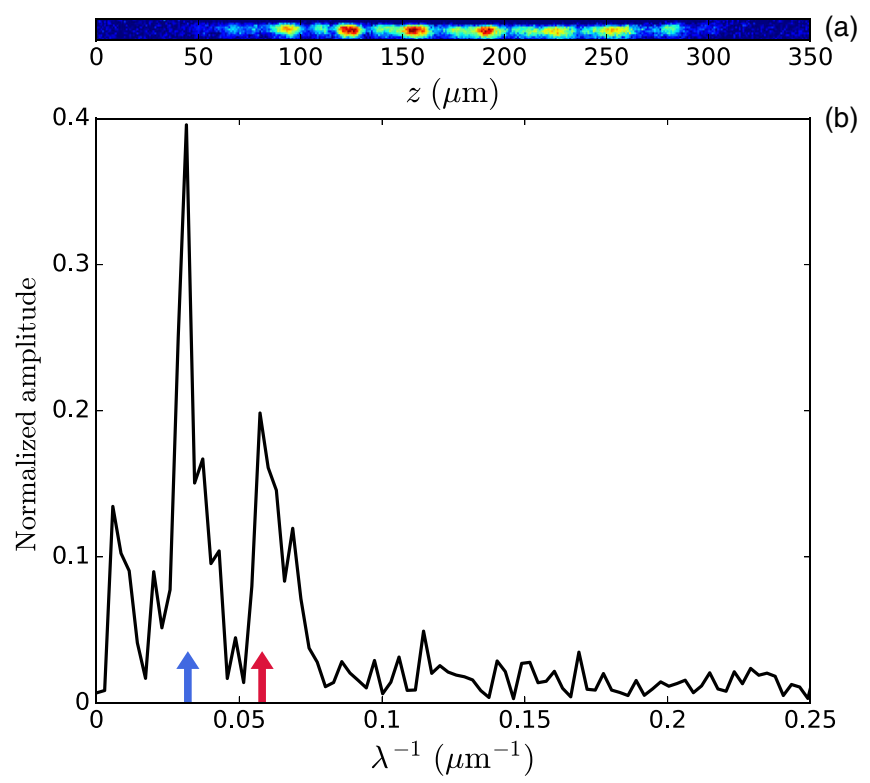

FIG. 3. (a) Image at $\omega=(2 \pi) 200 \mathrm{~Hz}$. (b) Spectrum showing the primary peak, which corresponds to $\lambda_{F}$, and the secondary peak due to the resonant mode. The blue and red arrows indicate the calculated values for $\lambda_{F}^{-1}$ and $\lambda_{R}^{-1}$, respectively, for these parameters. Here, $\Delta B=35 \mathrm{G}$, but, since $a(B)$ is a nonlinear function of $\Delta B$, the bounds $a_{+}=12 a_{0}$ and $a_{-}=-0.9 a_{0}$ are not symmetrically located about $\bar{a}=4.2 a_{0}$. Also, $t_{m}=t_{h}=20 \mathrm{~ms}$. 
We also explored a more impulsive regime, with short $t_{m}$ and where $\omega$ is kept within $10 \%$ of the Faraday resonance at $\omega=2 \omega_{r}$. In this case, with short $t_{m}$, we find that the wavelength of the resulting Faraday pattern is constant, independent of $\omega$.

The Faraday period also depends on the strength of the nonlinearity, as shown in Fig. 4, where both the measured and calculated [15] values of $\lambda_{F}$ are plotted vs the interaction parameter $\bar{a} \bar{\rho}$, where $\bar{\rho}$ is the line density obtained by integrating the column density along the transverse direction. The measured period is consistent with the 3D theory from Ref. [15].

We also explore the dynamics for the emergence of the Faraday pattern and its persistence following a short modulation time interval of $t_{m}=5 \mathrm{~ms}$ near $2 \omega_{r}$. Figure 5(a) shows the magnitude of the primary peak in the FFT spectrum vs $t_{h}$. Following modulation, the Faraday pattern forms after $t_{h} \simeq 20 \mathrm{~ms}$. By $t_{h}=50 \mathrm{~ms}$, however, the Faraday pattern vanishes before reemerging again at $t_{h} \simeq 90 \mathrm{~ms}$. A subsequent weaker collapse and revival occur at later $t_{h}$. We can gain some intuition as to the origins of this behavior by comparing measurements of the condensate length vs $t_{h}$. Figure 5(b) shows the axial Thomas-Fermi radius during the same $t_{h}$ interval. It shows

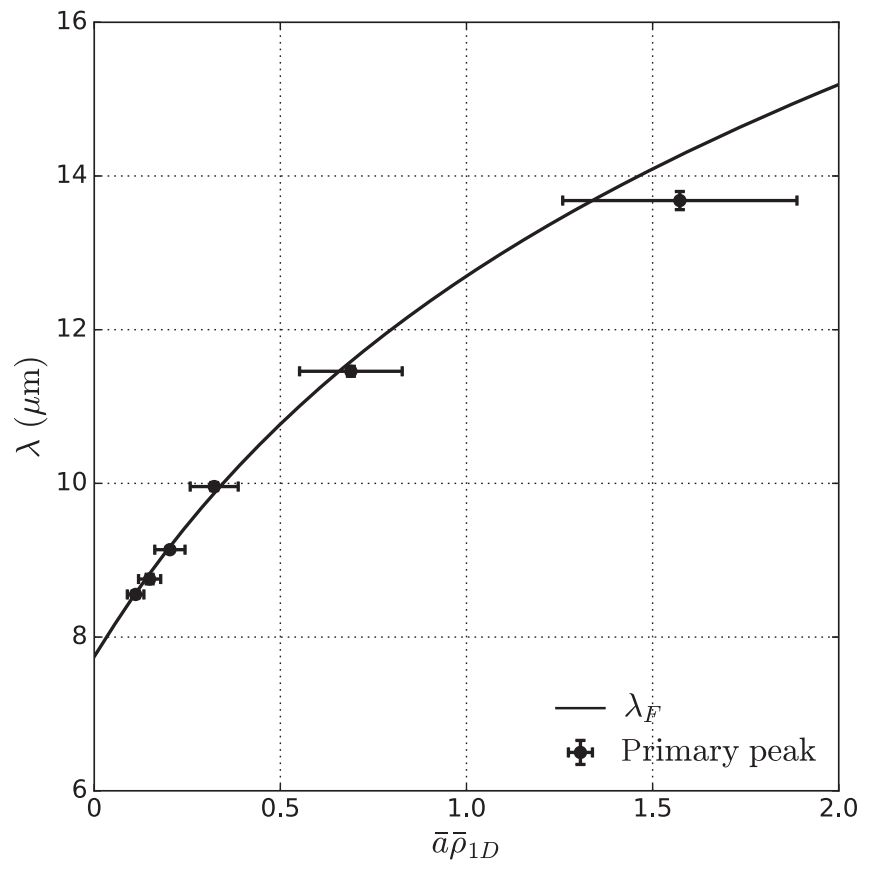

FIG. 4. Interaction dependence of $\lambda_{F}$. The relevant interaction parameter is $\bar{a} \bar{\rho}$, where $\bar{\rho}$ is the average line density and $\bar{a}$ varies between $1 a_{0}$ and $26 a_{0}$. Here, $\Delta B=5 \mathrm{G}$, corresponding to $\Delta a=$ $0.7 a_{0}$ for $\bar{a}=1 a_{0}$ and $\Delta a=3 a_{0}$ for $\bar{a}=26 a_{0}$. The data are indicated by filled squares, while the solid line is the theory of Ref. [15]. The error bars along the vertical axis correspond to the standard error, determined from ten different experimental runs, while the error bars along the horizontal axis arise from the systematic uncertainty in determining $\bar{a}$ [30].
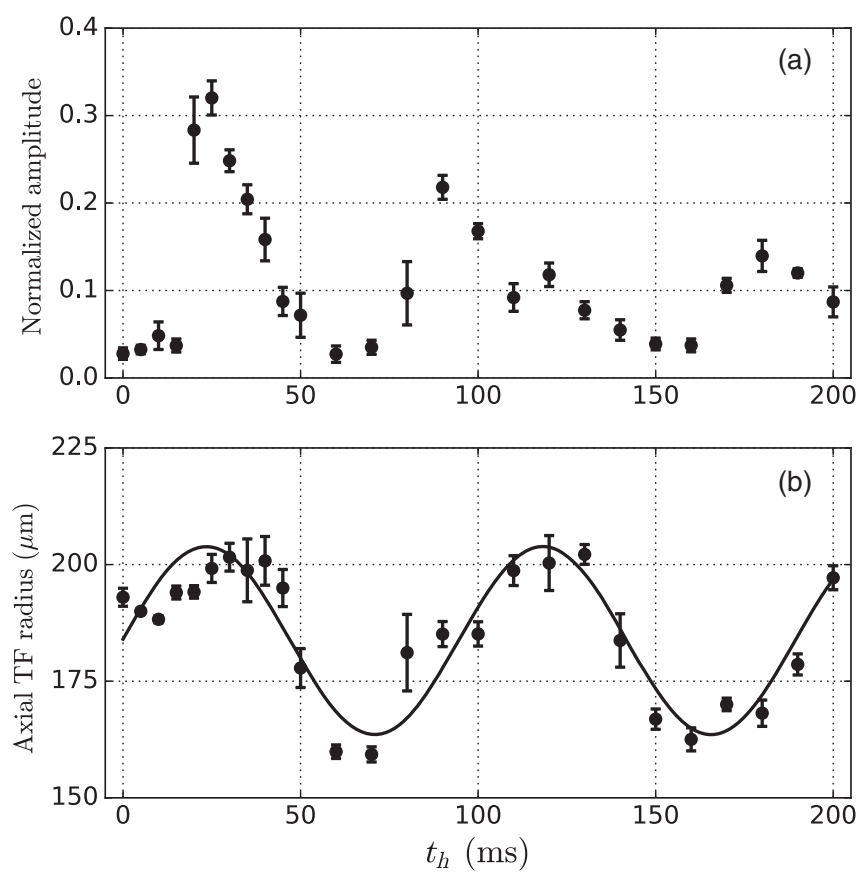

FIG. 5. Growth and suppression of the Faraday pattern. (a) The normalized amplitude of the primary spatial frequency in the FFT spectrum as a function of $t_{h}$. (b) The fitted axial Thomas-Fermi radius of the central region over the same time interval is shown by the filled circles. The solid line is a sinusoidal fit corresponding to a period of $95 \mathrm{~ms}$. For these data, $\omega=(2 \pi) 950 \mathrm{~Hz}$ and $t_{m}=5 \mathrm{~ms}$.

that a low-frequency collective mode is excited by the coupling to the modulated nonlinearity. The parameters of this condensate place it between the 1D mean-field and the 3D cigar regimes [35]. In the 3D Thomas-Fermi limit, the lowest $m=0$ quadrupolar mode for an elongated condensate has a frequency of $\sqrt{5 / 2} \omega_{z}$, while in the 1D limit the collective mode oscillates at $\sqrt{3} \omega_{z}$ [35-37]. For $\omega_{z}=(2 \pi) 7 \mathrm{~Hz}$, the corresponding period for this mode is, therefore, approximately $90 \mathrm{~ms}$, which is close to the observed oscillation period of $95 \mathrm{~ms}$. We find that the Faraday pattern is suppressed during axial compression but subsequently revives as the condensate returns to its original size. The phase of the two oscillations, the FFT amplitude and the Thomas-Fermi radius, do not exactly coincide. We attribute this observation to the delay in the initial growth of the Faraday pattern. We have determined experimentally that the frequency of the collapse and revival of the Faraday pattern scales with the axial trap frequency. A similar collapse and revival of the Faraday wave was previously observed [19].

\section{GRANULATION}

A Faraday pattern is not observed for low-frequency modulation, for which $\omega \ll \omega_{r}$. We find that, as $\omega$ is reduced, both modulation time $t_{m}$ and modulation 
amplitude $\Delta B$ must be increased in order to observe any change. As these parameters are increased, more spatial frequencies contribute (see Fig. 3), and, as $t_{m}$ and $\Delta B$ are increased further, we observe random patterns spanning a broad spatial frequency range, resembling grains [26,27]. We do not observe a significant thermal fraction before or after modulation, and therefore we attribute the observed granular patterns to quantum fluctuations and use a theory applicable to pure states.

In Fig. 6(a), we show experimental images and compare them to Gross-Pitaevskii (GP) simulations. Note that the axial and radial trap frequencies in this section are $\omega_{z}=$ $(2 \pi) 8 \mathrm{~Hz}$ and $\omega_{r}=(2 \pi) 254 \mathrm{~Hz}$, respectively. We observe that granulation is remarkably persistent in time after the modulation is turned off and that its structure is random between different experimental runs. GP simulations for similar parameters are shown in Fig. 6(b). In contrast to the experimental images, the GP simulations produce column-density distributions that resemble Faraday waves, with a regularly spaced pattern. Without a stochastic component, the GP model represents a crude approximation. The qualitative difference between the observations in Fig. 6(a) and the GP simulations in Fig. 6(b) suggests that the observed state of the atoms in the experiment goes beyond what the GP mean-field theory can describe.

The GP ansatz is a product of one single-particle state $\phi_{\mathrm{GP}}: \Psi_{\mathrm{GP}} \sim \prod_{k=1}^{N} \phi_{\mathrm{GP}}\left(r_{k}\right)$. This is a "mean-field state," because all particles in the many-body system occupy the single-particle state $\phi_{\mathrm{GP}}(r)$. A GP product state cannot describe correlations, where the properties of one or several particles in the many-body system depend on the properties of other particles in it. We go beyond the mean-field GP theory by employing the MCTDHB or MB, which can account for many-body correlations. The MCTDHB ansatz incorporates all possible configurations $\left(n_{1}, \ldots, n_{M}\right)$ of $N$ particles in $M$ single-particle states, $|\Psi\rangle=$ $\sum_{n_{1}, n_{2}, \ldots, n_{M}} C_{n_{1}, n_{2}, \ldots, n_{M}}\left|n_{1}, \ldots, n_{M}\right\rangle$. The MCTDHB ansatz can therefore self-consistently describe correlations in the many-body state [38].

We simulate the in situ single-shot images $[39,40]$ from the wave functions obtained with the MCTDHB for the various experimental parameters and for $M=2$ modes (see Supplemental Material in Ref. [38] and Refs. [41-49] therein). The simulated single-shot images correspond to drawing random samples from the $N$-particle density $\left|\Psi\left(r_{1}, \ldots, r_{N}\right)\right|^{2}$ of the many-body state. Single-shot images thus contain information about quantum fluctuations and correlation functions of all orders, and the average of many such single-shot images corresponds to the density. Because of computational constraints, at present, we can perform only 1D simulations. Along the axial direction, the experimental data show grains that are typically $4-10 \mu \mathrm{m}$ in length in the axial direction, while granulation is suppressed transversely, thus justifying the validity of the 1D approximation and our comparison of the 1D theory with the experimental line densities.

The simulation of single-shot images requires a model of the many-body probability distribution $\left|\Psi\left(r_{1}, \ldots, r_{N}\right)\right|^{2}$ as provided by the MCTDHB. Classical field methods, in contrast, approximate the time evolution of expectation values using "classical-field trajectories," i.e., solutions of the GP equation with stochastic initial conditions. These classical-field methods, however, do not supply a model for the wave function $\left|\Psi\left(r_{1}, \ldots, r_{n}\right)\right|^{2}$ from which single shots can be simulated [39].

Figure 7(a) shows the line density for three independent experimental shots and for four modulation frequencies, $\omega / 2 \pi=0,20,60$, and $80 \mathrm{~Hz}$, where $\omega=0$ corresponds to no modulation. For these data, the timescales $t_{m}=t_{h}=$ $250 \mathrm{~ms}$ are much longer than for the data discussed in the context of Faraday waves. The 1D MB simulations of the density and, for comparison to the experiment, two single $t_{h}(\mathrm{~ms})$

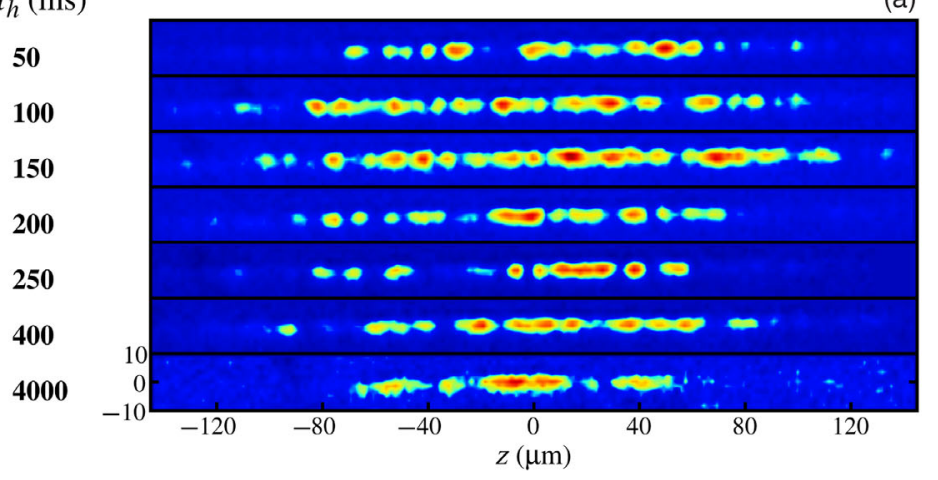

(a)

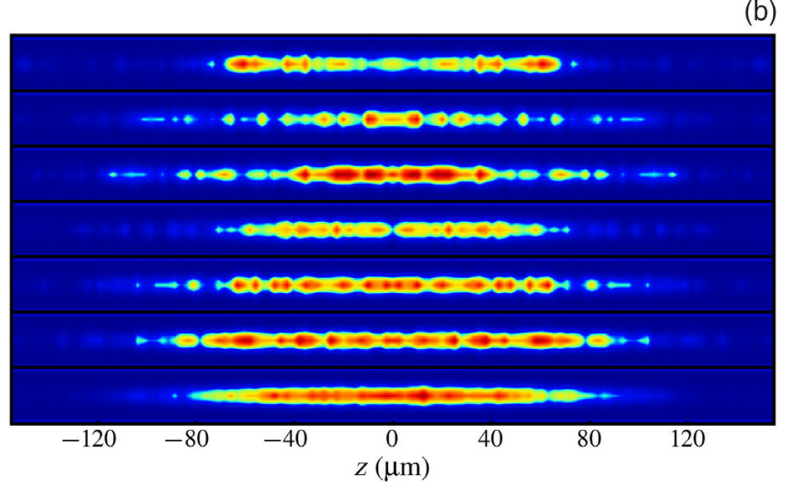

FIG. 6. (a) Experimental images and (b) GP simulations of column-density images for several values of $t_{h}$ and with $\omega=(2 \pi) 70 \mathrm{~Hz}$ and $t_{m}=250 \mathrm{~ms}$. The axial and radial trap frequencies for the experiments and simulations in this section are $\omega_{z}=(2 \pi) 8 \mathrm{~Hz}$ and $\omega_{r}=(2 \pi) 254 \mathrm{~Hz}$, respectively. (a) For the experiment, $\bar{B}=577.4 \mathrm{G}$ and $\Delta B=41.3 \mathrm{G}$, corresponding to $\bar{a}=5 a_{0}, a_{+}=15 a_{0}$, and $a_{-}=-1 a_{0}$. Each image, with indicated $t_{h}$, is a separate realization of the experiment. (b) Cylindrically symmetric 3D GP simulations where the calculated 3D densities are integrated along one transverse direction to produce 2D column densities. For the simulations, $a_{+}=20 a_{0}$ and $a_{-}=0.5 a_{0}$. 


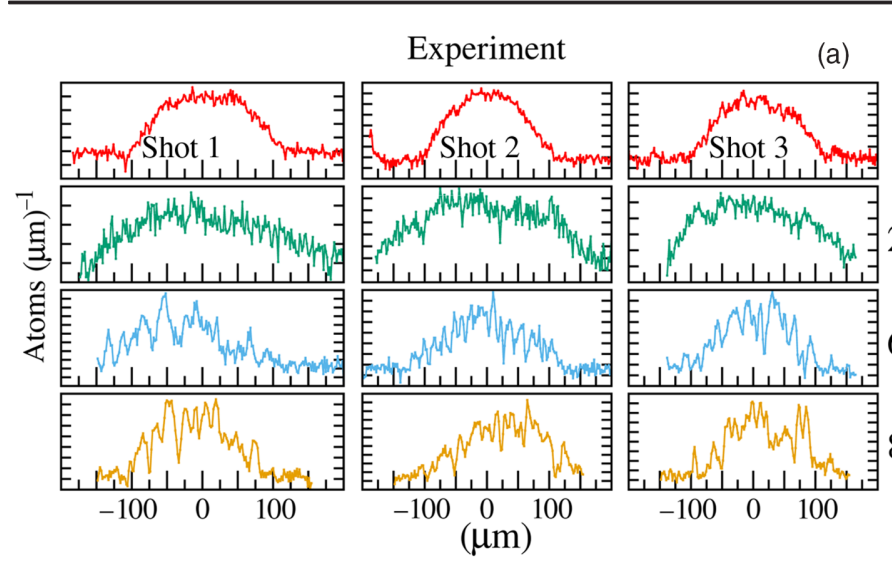

(a)

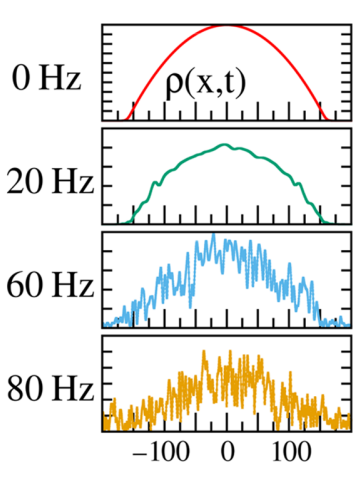

Theory

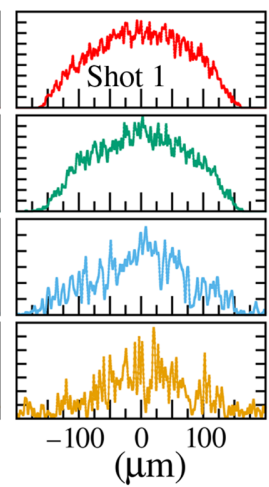

(b)

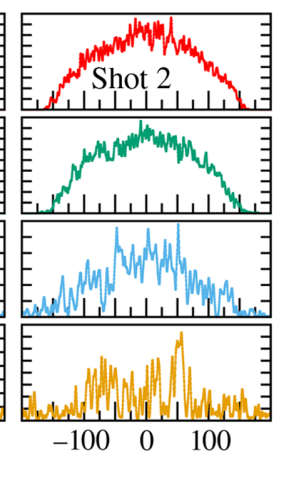

FIG. 7. Experimental and theoretical line-density profiles. (a) Experimental data and (b) many-body simulations for different modulation frequencies. (a) The rows show data for three independent experimental images ("shots") for the indicated $\omega$, where $\omega=0$ corresponds to no modulation. Here, $\bar{B}=590.8 \mathrm{G}, \Delta B=41.3 \mathrm{G}$, corresponding to $\bar{a}=8 a_{0}, a_{+}=20 a_{0}, a_{-}=0.7 a_{0}$, and $t_{m}=t_{h}=250 \mathrm{~ms}$. (b) The first column shows the density $\rho(z, t)$ as calculated from the 1D MB theory (see Methods), while the second and third columns display two simulated single shots. We observe that granulation is present in the single-shot images but absent in the average, $\rho(x, t)$.

shots are shown in Fig. 7(b). The single-shot simulations and experimental images are qualitatively similar, in contrast to the densities $\rho(x, t)$, obtained from the MB model. The shot-to-shot fluctuations in the single-shot simulations result from the fact that single shots are random samples distributed according to the many-body probability distribution $\left|\Psi\left(r_{1}, \ldots, r_{N} ; t\right)\right|^{2}$. At $\omega=(2 \pi) 20 \mathrm{~Hz}$, the experimental line density is somewhat broadened, perhaps indicating an excitation of low-lying quadrupolar oscillations. For $60 \mathrm{~Hz}$ modulation, the single-shot images exhibit large minima and maxima, which are even more pronounced at $80 \mathrm{~Hz}$. Thus, we find that there is a threshold modulation frequency $\omega_{c}$, above which the line density is significantly altered. The density, corresponding to the average of a large number of single shots, does not exhibit grains; they emerge only in single-shot images.

Figure 8 shows the second-order correlation functions for the experiment $C^{(2)}\left(z, z^{\prime}\right)$, and the MB theory $g^{(2)}\left(z, z^{\prime}\right)$, where both quantities are defined in Supplemental Material [38]. $C^{(2)}\left(z, z^{\prime}\right)$ are evaluated using an average of up to four experimental shots, whereas $g^{(2)}\left(z, z^{\prime}\right)$ are computed directly from the MCTDHB wave functions.

In both the experiment [Fig. 8(a)] and MB theory [Fig. 8(b)], we find that when $\omega<\omega_{c}$ the condensate is practically uncorrelated, as evidenced by $C^{(2)}\left(z, z^{\prime}\right) \approx$ $g^{(2)}\left(z, z^{\prime}\right) \approx 1$. However, when $\omega>\omega_{c}$, we find that the relatively constant correlation plane evolves into smaller correlated and anticorrelated regions, as shown Fig. 8(c) for the experiment and Fig. 8(d) for the MB theory.

To further characterize the granulated states, we plot the contrast parameter $\mathcal{D}$ at each modulation frequency in Fig. 9(a). $\mathcal{D}$ quantifies the deviation of a given set of single shots from a parabolic fit-as discussed in Supplemental Material [38] and Fig. S1 therein. A sharp threshold can be
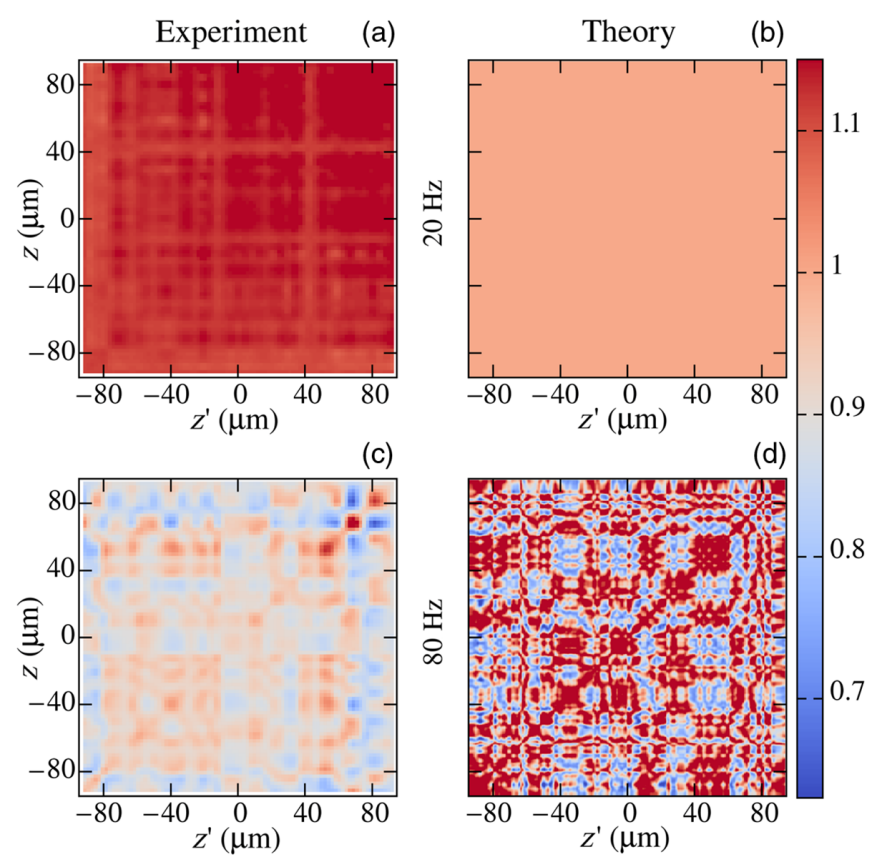

FIG. 8. Second-order correlation functions. (a) Correlation function $C^{(2)}\left(z, z^{\prime}\right)$ calculated from the experimental data for $\omega=(2 \pi) 20 \mathrm{~Hz}$. (b) Correlation function $g^{(2)}\left(z, z^{\prime}\right)$ calculated from the MB theory for the same parameters as (a). (c) $C^{(2)}\left(z, z^{\prime}\right)$ calculated from the experimental data for $\omega=(2 \pi) 80 \mathrm{~Hz}$. (d) $g^{(2)}\left(z, z^{\prime}\right)$ calculated from the MB theory for the same parameters as (c). For the nongranulated states $[(a),(b)]$, the correlation function is approximately 1 , indicating the absence of second-order correlations. For the granulated states [(c),(d)], regions with correlations (red hues) and anticorrelations (blue hues) emerge. Theoretical and experimental second-order correlations qualitatively agree: They are flat for the nongranular states [(a),(b)] and exhibit patterns of comparable length scale and magnitude for the granular states [(c),(d)]. All images correspond to $t_{h}=t_{m}=250 \mathrm{~ms}$ and $\bar{a}=8 a_{0}, a_{+}=20$, and $a_{-}=0.5 a_{0}$. 
seen in both the experimental data and the simulations at $\omega_{c} \approx(2 \pi) 30 \mathrm{~Hz}$, beyond which grains start to form. For $\omega<\omega_{c}$, the gas oscillates coherently without a significant deviation from a Thomas-Fermi envelope.

The threshold frequency $\omega_{c}$ can be understood by examining the second-largest eigenvalues (or occupations) $n_{2}^{(1)}$ and $n_{2}^{(2)}$ of the first- and second-order reduced density matrices (RDMs), respectively (see Methods [38]), which are plotted in Fig. 9(b). These may be used as a measure of the departure of our MB model from mean-field states. Many-body systems, where multiple eigenvalues of the first-order RDM are macroscopic (i.e., of the order of $N$ ),

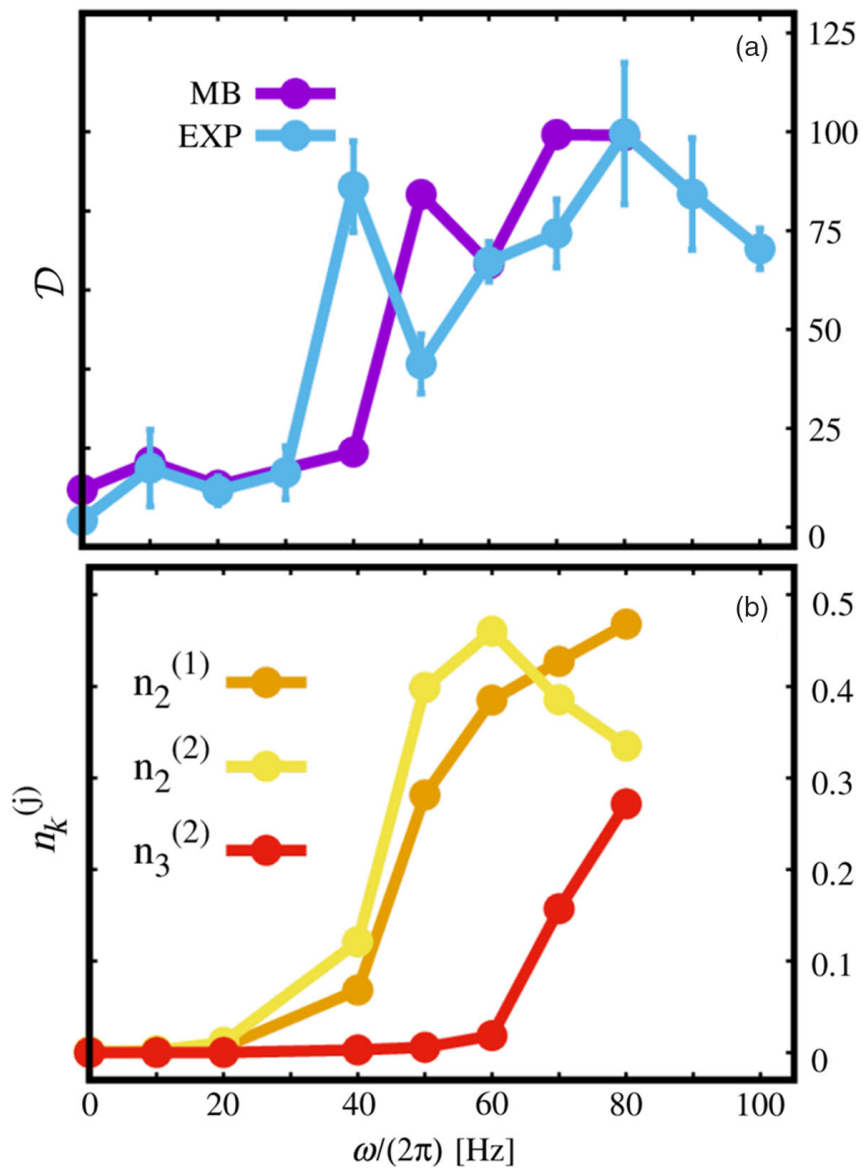

FIG. 9. Granulation vs $\omega$. (a) Comparison of the deviations from a Thomas-Fermi distribution as quantified by the contrast parameter $\mathcal{D}=\mathcal{D}(\omega)$ [38] for single shots simulated with the MB theory with those taken in the experiment (EXP). The MB theory predicts the threshold value $\omega_{c} \approx(2 \pi) 30 \mathrm{~Hz}$, where deviations become large and grains form. Each symbol and its error bar are the mean and standard error, respectively, of the mean of at least four experimental measurements of $\mathcal{D}$, while 100 single shots at each $\omega$ are used for the MB simulations. (b) Eigenvalues of the first- and second-order RDM. The growth of all three is observed to occur for $\omega>\omega_{c}$, indicating the emergence of correlations and fragmentation. The growth of both $n_{2}^{(1)}$ and $n_{2}^{(2)}$ occur as $\omega \approx \omega_{c}$, with the drop in $n_{2}^{(2)}$ near $60 \mathrm{~Hz}$ corresponding to the subsequent growth in $n_{3}^{(2)}$. are termed fragmented [47,50]. At zero excitation, only $n_{1}^{(1),(2)}$ are macroscopic, while $n_{2}^{(1),(2)}$ are nearly zero. The latter increase substantially with $\omega$ beyond $\omega_{c}$, heralding the loss of first- and second-order coherence and the emergence of correlations as shown in Fig. 8. At $\omega \approx(2 \pi) 50 \mathrm{~Hz}$, we observe a drop in $n_{2}^{(2)}$, which, however, results in an increase in $n_{3}^{(2)}$ and not an increase in $n_{1}^{(1),(2)}$. The MCTDHB computations thus show that the emergence of granulation is accompanied by the conversion of initial condensation (only a single macroscopic occupation [45]) into fragmentation.

Both observations, the emergence of fragmentation and the loss of second-order coherence, underscore that the granulation of Bose-Einstein condensates is a many-body effect. The system thus cannot be described by a mean-field product state any longer and has left the realm of GP theory. Although the transition to fragmentation is not sharp-the natural occupations $n_{i}^{(1),(2)}$ take on continuous values-it is well established at sufficiently large $\omega$. Granulation features randomly distributed variably sized grains of atoms which can be observed in single-shot images. Fragmentation, or depletion, on the other hand, is characterized by the reduced density matrix and its (macroscopic) eigenvalues and is not necessarily accompanied by granulation of the density $[47,50]$. In our close-to-onedimensional setup, we observe granulation to emerge side by side with fragmentation.

The dynamical evolution, as calculated from the MB theory, of the density is shown in Figs. 10(a) and 10(d) for $\omega<\omega_{c}$ and $\omega>\omega_{c}$, respectively. In both cases, the modulation of the Thomas-Fermi radius follows the external perturbation. Once the modulation is turned off, the radius oscillates at its natural quadrupolar frequency. The first-order spatial coherence is shown in Figs. 10(b) and $10(\mathrm{e})$ for the same parameters. The patterns that emerge and persist in $g^{(1)}\left(z, z^{\prime}\right)$ demonstrate that spatial correlations between particles at distinct and distant locations in the granular state are present [Fig. 10(e)]. The length scale of the patterns in $g^{(1)}\left(z, z^{\prime}\right)$ is similar to what is seen in Fig. 8 for $g^{(2)}\left(z, z^{\prime}\right)$. We infer that the process of granulation in a BEC is accompanied by the emergence of nonlocal correlations in the many-body state. Figure 10(f) shows the emergence of two macroscopic eigenvalues of the reduced one-body density matrix for $\omega>\omega_{c}$. While these so-called natural occupations are unaffected by modulation for $\omega<\omega_{c}$, as seen in Fig. 10(c), $\omega>\omega_{c}$ results in the second natural orbital being macroscopically populated and, hence, in the fragmentation of the BEC [Fig. 10(f)]. An examination of the total energy per particle $\left(E_{t}\right)$ imparted during modulation for a time $t_{m}$ shows that $E_{t} \approx 22 \mathrm{nK}$ when $\omega=(2 \pi) 20 \mathrm{~Hz}$ and $E_{t} \approx 36 \mathrm{nK}$ when $\omega=(2 \pi) 80 \mathrm{~Hz}$, both of which are much less than the critical temperature $T_{c} \approx 330 \mathrm{nK}$. 


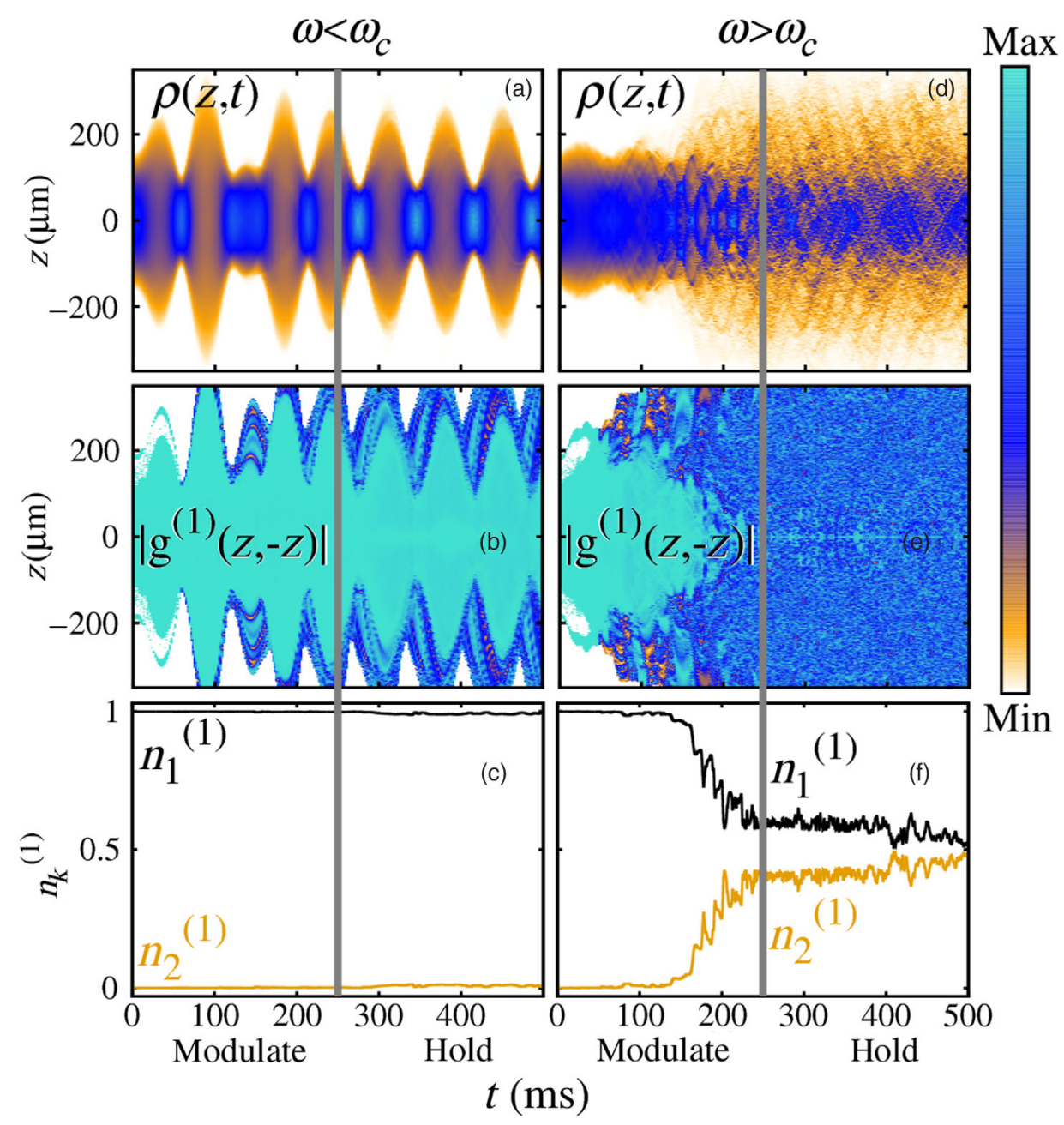

FIG. 10. Time evolution, coherence, and fragmentation from simulations. (a),(d) The density $\rho(z, t)$, (b),(e) first-order spatial correlation function $\left|g^{(1)}(z,-z)\right|$, and (c),(f) natural occupations $n_{k}^{(1)}(t)$ are plotted vs time $t . n_{1}^{(1)}$ is denoted by the black line, while $n_{2}^{(1)}$ is indicated by the yellow line. (a)-(c) are calculated with $\omega=(2 \pi) 20 \mathrm{~Hz}<\omega_{c}$ and (d)-(f) with $\omega=(2 \pi) 80 \mathrm{~Hz}>\omega_{c}$. All other parameters are given in the caption of Fig. 7. The onset and formation of granulation are inferred by the simultaneous drop in the values of $\left|g^{(1)}\right|$ and $n_{1}^{(1)}$, indicating the emergence of spatial correlations and fragmentation, respectively.

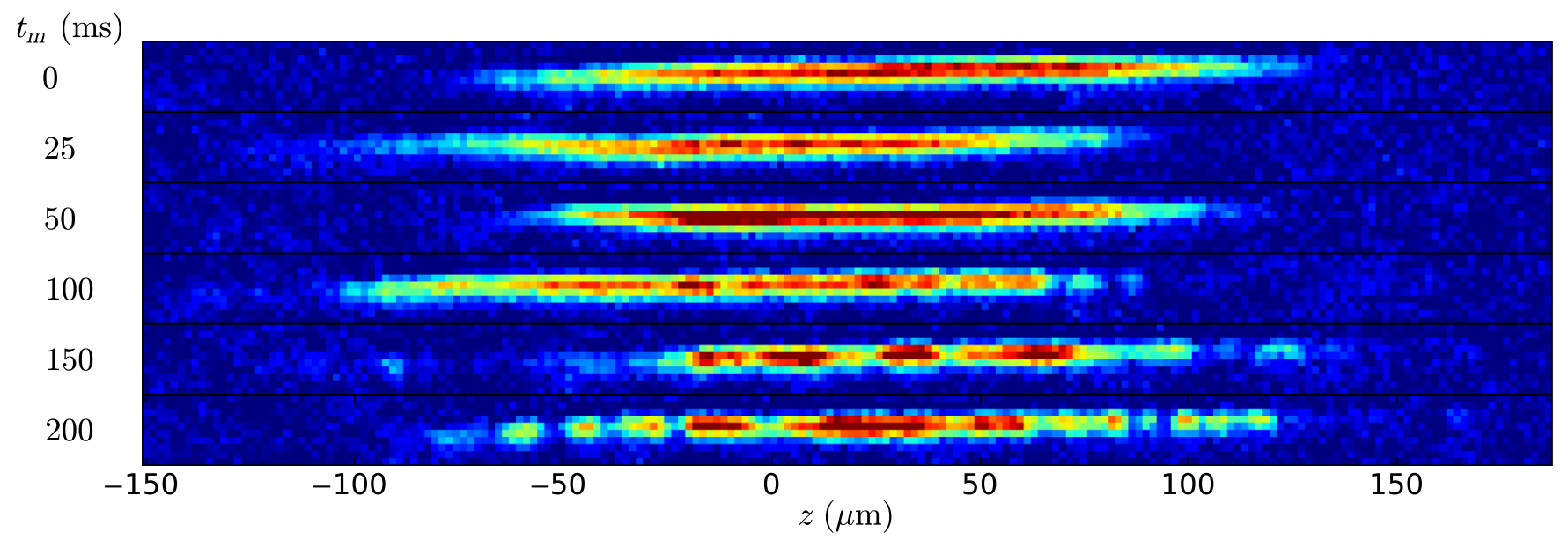

FIG. 11. Experimental column densities showing the formation of grains. Representative column-density images taken at different $t_{m}$. For each value of $t_{m}, \omega=(2 \pi) 70 \mathrm{~Hz}$ and $t_{h}=250 \mathrm{~ms}$. All other parameters are given in the caption of Fig. 7. Each image is a different realization of the experiment. 
The onset of granulation observed experimentally is shown in Fig. 11. The condensate was modulated at $\omega=$ $(2 \pi) 70 \mathrm{~Hz}$ for various $t_{m}$ followed by $t_{h}=250 \mathrm{~ms}$. For $t_{m}<100 \mathrm{~ms}$, there is no discernable difference between the modulated and unmodulated $\left(t_{m}=0 \mathrm{~ms}\right)$ cases, but for $t_{m}>100 \mathrm{~ms}$ grains are observed to form. Consistent with Fig. 10, the transition to a granulated state is gradual with increasing $t_{m}$. The observed grains are also long-lived in comparison to Faraday waves, as shown in Figs. 6(a) and 5(a), respectively.

The transition to granular states occurs due to the presence of quantum correlations. The second-order correlations, shown in Fig. 8, and the first-order, nonlocal correlations, shown in the middle panel in Fig. 10, result from modulating the interaction and do not disappear after the modulation is stopped. Our modeling of the state on the many-body level suggests that granulation represents a dynamical many-body state characterized by the presence of quantum fluctuations, correlations, fragmentation, and their persistence in time.

Granular states feature random patterns and lack periodicity in their distributions, distinguishing them from Faraday and shock waves [51]. The multicharacteristic nature of quantum grains is supported by our observation of additional anomalous features in real and momentum space. Indeed, we find signatures of different coexisting phases of perturbed quantum systems such as quantum turbulence and localization in granulated states. We verified that the density in momentum space (as calculated from the MB theory) of the granulated state shows clear signs of a $k^{-2}$ power-law scaling (see Supplemental Material [38] and Fig. S2 therein), which indicates a connection to turbulent BECs [52-55].

\section{CONCLUSIONS}

We have explored the response of a BEC to modulated interactions. In the regime where the drive frequency $\omega \gtrsim \omega_{r}$, the drive couples to parametric and resonant modes that result in one-dimensional spatial pattern formation. For $\omega$ near resonance with $2 \omega_{r}$ or $\omega_{r}$, very little modulation time and amplitude are required to produce a significant response. Near these resonances, the condensate undergoes breathing oscillations that persist for a long time, resulting in the formation of Faraday and resonant mode patterns for $t_{h}>0$. A pattern is also observed off resonance, but only with an increased modulation amplitude and modulation time. Because of the long modulation time, the resulting pattern can be seen at $t_{h}=0$ and is a direct consequence of the applied modulation. The dispersion relation of both Faraday and resonant modes is well represented by a meanfield theory that accounts for the three-dimensional nature of the elongated condensate.

For lower drive frequencies, the modulated interactions only weakly couple to the condensate. Significant response is achieved only by increasing the modulation amplitude and time and then only above a critical modulation frequency $\omega_{c}$.
Fluctuating and irregular spatial patterns, that we define as grains, may then emerge and persist for long periods of time. A theoretical description of granulation requires approaches that go beyond the mean-field theory, indicating that quantum granulation is characterized by nonlocal manybody correlations and quantum fluctuations.

\section{ACKNOWLEDGMENTS}

This work was supported in part by the Army Research Office Multidisciplinary University Research Initiative (Grant No. W911NF-14-1-0003), the Office of Naval Research, the National Science Foundation (Grant No. PHY-1707992), the Welch Foundation (Grant No. C-1133), the Austrian Science Foundation (FWF) under Grants No. F41(SFB "ViCoM") and No. P32033, the Wiener Wissenschafts- und TechnologieFonds (WWTF) Project No. MA16-066 ("SEQUEX"), and by FAPESP, under the CEPID program (Grant No. 2013/07276-1). Computational time in the High-Performance Computing Center Stuttgart (HLRS) is gratefully acknowledged. We also thank Mustafa Amin for valuable discussions.

[1] M. C. Cross and P.C. Hohenberg, Pattern Formation Outside of Equilibrium, Rev. Mod. Phys. 65, 851 (1993).

[2] M. Faraday, Xvii. On a Peculiar Class of Acoustical Figures; and on Certain Forms Assumed by Groups of Particles upon Vibrating Elastic Surfaces, Phil. Trans. R. Soc. London 121, 299 (1831).

[3] S. Douady and S. Fauve, Pattern Selection in Faraday Instability, Europhys. Lett. 6, 221 (1988).

[4] R. Keolian, L. A. Turkevich, S. J. Putterman, I. Rudnick, and J. A. Rudnick, Subharmonic Sequences in the Faraday Experiment: Departures from Period Doubling, Phys. Rev. Lett. 47, 1133 (1981).

[5] S. Ciliberto and J. P. Gollub, Pattern Competition Leads to Chaos, Phys. Rev. Lett. 52, 922 (1984).

[6] S. Ciliberto, S. Douady, and S. Fauve, Investigating SpaceTime Chaos in Faraday Instability by Means of the Fluctuations of the Driving Acceleration, Europhys. Lett. 15, 23 (1991).

[7] M. J. Feigenbaum, The Onset Spectrum of Turbulence, Phys. Lett. A 74, 375 (1979).

[8] T. B. Benjamin and F. Ursell, The Stability of the Plane Free Surface of a Liquid in Vertical Periodic Motion, Proc. R. Soc. A 225, 505 (1954).

[9] J. Bechhoefer and B. Johnson, A Simple Model for Faraday Waves, Am. J. Phys. 64, 1482 (1996).

[10] J. J. García-Ripoll, V. M. Pérez-García, and P. Torres, Extended Parametric Resonances in Nonlinear Schrödinger Systems, Phys. Rev. Lett. 83, 1715 (1999).

[11] K. Staliunas, S. Longhi, and G. J. de Valcárcel, Faraday Patterns in Bose-Einstein Condensates, Phys. Rev. Lett. 89, 210406 (2002).

[12] K. Staliunas, S. Longhi, and G. J. de Valcárcel, Faraday Patterns in Low-Dimensional Bose-Einstein Condensates, Phys. Rev. A 70, 011601 (2004). 
[13] A. I. Nicolin, R. Carretero-González, and P. G. Kevrekidis, Faraday Waves in Bose-Einstein Condensates, Phys. Rev. A 76, 063609 (2007).

[14] R. Nath and L. Santos, Faraday Patterns in TwoDimensional Dipolar Bose-Einstein Condensates, Phys. Rev. A 81, 033626 (2010).

[15] A. I. Nicolin, Resonant Wave Formation in Bose-Einstein Condensates, Phys. Rev. E 84, 056202 (2011).

[16] A. Balaž, R. Paun, A. I. Nicolin, S. Balasubramanian, and R. Ramaswamy, Faraday Waves in Collisionally Inhomogeneous Bose-Einstein Condensates, Phys. Rev. A 89, 023609 (2014).

[17] H. Abe, T. Ueda, M. Morikawa, Y. Saitoh, R. Nomura, and Y. Okuda, Faraday Instability of Superfluid Surface, Phys. Rev. E 76, 046305 (2007).

[18] P. Engels, C. Atherton, and M. A. Hoefer, Observation of Faraday Waves in a Bose-Einstein Condensate, Phys. Rev. Lett. 98, 095301 (2007).

[19] A. Groot, Excitations in Hydrodynamic Ultra-cold Bose Gases, Ph.D. thesis, Utrecht University, 2015.

[20] L. W. Clark, A. Gaj, L. Feng, and C. Chin, Collective Emission of Matter-Wave Jets from Driven Bose-Einstein Condensates, Nature (London) 551, 356 (2017).

[21] B. A. Malomed, Soliton Management in Periodic Systems (Springer, New York, 2006).

[22] S. E. Pollack, D. Dries, R. G. Hulet, K. M. F. Magalhães, E. A. L. Henn, E. R. F. Ramos, M. A. Caracanhas, and V. S. Bagnato, Collective Excitation of a Bose-Einstein Condensate by Modulation of the Atomic Scattering Length, Phys. Rev. A 81, 053627 (2010).

[23] I. Vidanović, A. Balaž, H. Al-Jibbouri, and A. Pelster, Nonlinear Bose-Einstein-Condensate Dynamics Induced by a Harmonic Modulation of the s-Wave Scattering Length, Phys. Rev. A 84, 013618 (2011).

[24] H. M. Jaeger, S. R. Nagel, and R. P. Behringer, Granular Solids, Liquids, and Gases, Rev. Mod. Phys. 68, 1259 (1996).

[25] Granular Matter: An Interdisciplinary Approach, edited by A. Mehta (Springer-Verlag, New York, 1994).

[26] V. I. Yukalov, A. N. Novikov, and V. S. Bagnato, Formation of Granular Structures in Trapped Bose-Einstein Condensates under Oscillatory Excitations, Laser Phys. Lett. 11, 095501 (2014).

[27] V. I. Yukalov, A. N. Novikov, and V. S. Bagnato, Realization of Inverse Kibble-Zurek Scenario with Trapped Bose Gases, Phys. Lett. A 379, 1366 (2015).

[28] A. I. Streltsov, O. E. Alon, and L. S. Cederbaum, Role of Excited States in the Splitting of a Trapped Interacting Bose-Einstein Condensate by a Time-Dependent Barrier, Phys. Rev. Lett. 99, 030402 (2007).

[29] O. E. Alon, A. I. Streltsov, and L. S. Cederbaum, Multiconfigurational Time-Dependent Hartree Method for Bosons: Many-Body Dynamics of Bosonic Systems, Phys. Rev. A 77, 033613 (2008).

[30] S. E. Pollack, D. Dries, M. Junker, Y. P. Chen, T. A. Corcovilos, and R. G. Hulet, Extreme Tunability of Interactions in a ${ }^{7} \mathrm{Li}$ Bose-Einstein Condensate, Phys. Rev. Lett. 102, 090402 (2009).

[31] N. Gross, Z. Shotan, O. Machtey, S. Kokkelmans, and L. Khaykovich, Study of Efimov Physics in Two Nuclear-Spin Sublevels of 7Li, C.R. Phys. 12, 4 (2011).
[32] N. Navon, S. Piatecki, K. Günter, B. Rem, T. C. Nguyen, F. Chevy, W. Krauth, and C. Salomon, Dynamics and Thermodynamics of the Low-Temperature Strongly Interacting Bose Gas, Phys. Rev. Lett. 107, 135301 (2011).

[33] P. Dyke, S. E. Pollack, and R. G. Hulet, FiniteRange Corrections near a Feshbach Resonance and Their Role in the Efimov Effect, Phys. Rev. A 88, 023625 (2013).

[34] C. C. Bradley, C. A. Sackett, and R. G. Hulet, Bose-Einstein Condensation of Lithium: Observation of Limited Condensate Number, Phys. Rev. Lett. 78, 985 (1997).

[35] C. Menotti and S. Stringari, Collective Oscillations of a One-Dimensional Trapped Bose-Einstein Gas, Phys. Rev. A 66, 043610 (2002).

[36] S. Stringari, Collective Excitations of a Trapped BoseCondensed Gas, Phys. Rev. Lett. 77, 2360 (1996).

[37] M.-O. Mewes, M. R. Andrews, N. J. van Druten, D. M. Kurn, D. S. Durfee, C. G. Townsend, and W. Ketterle, Collective Excitations of a Bose-Einstein Condensate in a Magnetic Trap, Phys. Rev. Lett. 77, 988 (1996).

[38] See Supplemental Material at http://link.aps.org/ supplemental/10.1103/PhysRevX.9.011052 for experimental details and numerical methods used.

[39] K. Sakmann and M. Kasevich, Single-Shot Simulations of Dynamic Quantum Many-Body Systems, Nat. Phys. 12, 451 (2016).

[40] A. U. J. Lode and C. Bruder, Fragmented Superradiance of a Bose-Einstein Condensate in an Optical Cavity, Phys. Rev. Lett. 118, 013603 (2017).

[41] A. U. J. Lode, Multiconfigurational Time-Dependent Hartree Method for Bosons with Internal Degrees of Freedom: Theory and Composite Fragmentation of Multicomponent Bose-Einstein Condensates, Phys. Rev. A 93, 063601 (2016).

[42] E. Fasshauer and A. U. J. Lode, Multiconfigurational TimeDependent Hartree Method for Fermions: Implementation, Exactness, and Few-Fermion Tunneling to Open Space, Phys. Rev. A 93, 033635 (2016).

[43] A. U. J. Lode, M. C. Tsatsos, E. Fasshauer, R. Lin, L. Papariello, P. Molignini, C. Lévêque, and S. E. Weiner, MCTDH-X: The Time-Dependent Multiconfigurational Hartree for Indistinguishable Particles Software, http:// ultracold.org (2019).

[44] I. Březinová, A. U. J. Lode, A. I. Streltsov, O. E. Alon, L. S. Cederbaum, and J. Burgdörfer, Wave Chaos as Signature for Depletion of a Bose-Einstein Condensate, Phys. Rev. A 86, 013630 (2012).

[45] K. Sakmann, A. I. Streltsov, O. E. Alon, and L. S. Cederbaum, Reduced Density Matrices and Coherence of Trapped Interacting Bosons, Phys. Rev. A 78, 023615 (2008).

[46] O. Penrose and L. Onsager, Bose-Einstein Condensation and Liquid Helium, Phys. Rev. 104, 576 (1956).

[47] R. W. Spekkens and J. E. Sipe, Spatial Fragmentation of a Bose-Einstein Condensate in a Double-Well Potential, Phys. Rev. A 59, 3868 (1999).

[48] I. Bouchoule, M. Arzamasovs, K. V. Kheruntsyan, and D. M. Gangardt, Two-Body Momentum Correlations in a Weakly Interacting One-Dimensional Bose Gas, Phys. Rev. A 86, 033626 (2012). 
[49] G. Roati, C. D’Errico, L. Fallani, M. Fattori, C. Fort, M. Zaccanti, G. Modugno, M. Modugno, and M. Inguscio, Anderson Localization of a Non-Interacting Bose-Einstein Condensate, Nature (London) 453, 895 (2008).

[50] E. J. Mueller, T.-L. Ho, M. Ueda, and G. Baym, Fragmentation of Bose-Einstein Condensates, Phys. Rev. A 74, 033612 (2006).

[51] V. M. Pérez-García, V. V. Konotop, and V. A. Brazhnyi, Feshbach Resonance Induced Shock Waves in BoseEinstein Condensates, Phys. Rev. Lett. 92, 220403 (2004).

[52] K. J. Thompson, G. G. Bagnato, G. D. Telles, M. A. Caracanhas, F.E. A. dos Santos, and V.S. Bagnato, Evidence of Power Law Behavior in the Momentum
Distribution of a Turbulent Trapped Bose-Einstein Condensate, Laser Phys. Lett. 11, 015501 (2014).

[53] N. Navon, A. L. Gaunt, R. P. Smith, and Z. Hadzibabic, Emergence of a Turbulent Cascade in a Quantum Gas, Nature (London) 539, 72 (2016).

[54] M. C. Tsatsos, P. E. S. Tavares, A. Cidrim, A. R. Fritsch, M. A. Caracanhas, F. E. A. dos Santos, C. F. Barenghi, and V.S. Bagnato, Quantum Turbulence in Trapped Atomic Bose-Einstein Condensates, Phys. Rep. 622, 1 (2016).

[55] P. E. S. Tavares, A. R. Fritsch, G. D. Telles, M. S. Hussein, F. Impens, R. Kaiser, and V.S. Bagnato, Matter Wave Speckle Observed in an Out-of-Equilibrium Quantum Fluid, Proc. Natl. Acad. Sci. U.S.A. 114, 12691 (2017). 